\title{
Effect of Leeway and Drift Angle of Ship Navigation and Determination Method
}

\author{
Haisheng Xiong ${ }^{1, a}$, Xiaolin Wang ${ }^{2}$ \\ ${ }^{1}$ Tianjin Maritime College, Haihe education park, Tianjin, China \\ ${ }^{2}$ Wuhan Maritime Vocation College, Shekou Street, Huangpi District, Wuhan City, Hubei Province China
}

\begin{abstract}
The wind and the current is an important factor affecting the safety of navigation of ships at sea. This article focuses on the influence of wind and flow of ships sailing and Predicted drift angle of dead reckoning tracer method, which plays an active role in ensuring the safety of navigation, and plays a guiding role in the determination of the leeway and drift angle for the staff on board and in solving the problems of students in navigation schools.
\end{abstract}

\section{Foreword}

In order to ensure the safety of navigation and make the ship sail according to the planned route, it is the key to measure the leeway and drift angle correctly. The measurement of leeway and drift angle should be based on the characteristics of navigation environment, so that the work of air pressure difference can be completed accurately and efficiently.

\section{Wind and current factors affecting ship navigation}

\subsection{Influence of wind on ship navigation}

\subsubsection{The classification of wind}

The wind is due to air flow generated by the wind vector, has both magnitude and direction, the direction of the wind is the direction of the wind blows. In windless weather, when a ship is sailing, because the ship has relative speed with the air, it will also have the feeling of wind. This kind of wind is called ship wind. The characteristic of ship wind is that the speed is the same as the speed of the ship, and the direction of the wind is the same as that of the ship. In windy weather, the wind measured by the ship's anemometer is the result of the combined action of the ship's wind and the true wind, which is called the apparent wind. In a word, the true wind can not be measured directly when a ship is sailing. It can only be calculated according to the triangle rule through the vector relationship among ship wind, sight wind and true wind.

$$
\overrightarrow{\text { apparent wind }}=\underset{\text { ship wind }}{\longrightarrow}+\underset{\text { true wind }}{\longrightarrow}
$$

The calculation method can be directly calculated by software, and can also be drawn by drawing method. The calculation principle is shown in Fig. 1. Firstly, the ship's wind vector is drawn along the opposite direction of the ship's course (the size is the same as the ship's speed and the wind direction is opposite to the course), then the apparent wind vector is drawn according to the measurement results of the ship's anemometer, and then the true wind vector is drawn by connecting the end of the ship's wind vector with the end of the apparent wind vector, and the vector direction represents the true wind direction. The length represents the true wind speed.

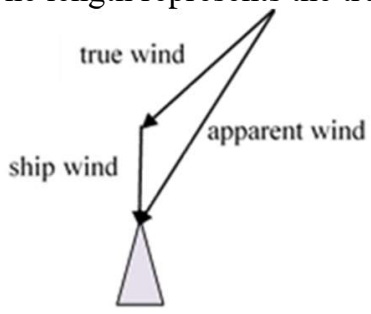

Figure 1. Principle of True Wind Computation.

\subsubsection{The wind effects on ship navigation}

When the wind direction is not in the same line with the ship's fore and aft lines, an angle is generated. The angle is called the relative bearing of wind $(\mathrm{QW})$, as shown in Figure 2. When the wind angle is close to 90 degrees, the ship's yaw is the most serious and the greater the wind pressure angle is. The wind deflected the ship's course to the right, and the wind pressure difference angle was

\footnotetext{
${ }^{a}$ Corresponding author: Haisheng Xiong email:820399665@qq.com
} 
positive, making the course deviation to the left, and the wind pressure difference angle was negative.

The empirical formula of wind pressure difference is obtained based on the measured data.

According to the measured statistics obtained leeway formula:

$$
\alpha=K^{0}\left(\frac{V_{W}}{V_{L}}\right)^{1.4}\left(\sin Q_{W}+0.15 \sin 2 Q_{W}\right)
$$

In the formula:

$\mathrm{V}_{\mathrm{L}} \mathrm{V}_{\mathrm{W}}-$ ship's speed、wind's speed $(\mathrm{m} / \mathrm{s})$;

$\mathrm{Q}_{\mathrm{W}}$ - relative bearing of wind;

$\mathrm{K}^{0}$ — leeway coefficient

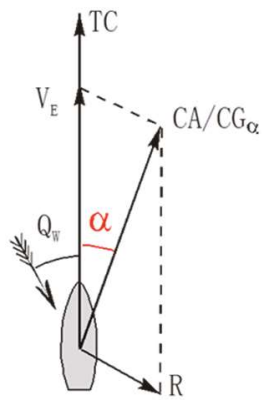

Figure 2. Diagram of leeway.

As shown in Fig. 2, the formula of the ship's track direction under the influence of wind is as follows:

$$
C G(C A)=C A_{\alpha}=T C \pm \alpha
$$

In the formula:

CG(CA)__ course made good(course of advance); $\mathrm{CG}_{\alpha}-$ course made good in wind;

TC_- ture course;

$\alpha$-leeway

\subsection{Influence of wind on ship navigation}

\subsubsection{The classification of current}

Ships sailing in the ocean are mainly affected by wind currents and ocean currents, and tidal currents should also be considered in coastal navigation. However, currents in reef areas and Straits mainly take into account the role of tidal currents, and their amount is also large. Ship pilots should pay special attention to the effects of currents on ships when navigating in the ocean, coastal, Straits and reef areas.

1) Wind Current

Wind currents are much stronger than other currents, so they are one of the currents that have the greatest impact on ship navigation in the ocean. Because of the action of sea surface wind and sea surface, the sea current that causes the sea water to flow horizontally is called wind current. When the wind direction remains unchanged for a certain period of time (usually more than 6 hours) and continues to blow across the sea surface, the sea surface will be affected by wind shear stress. Under the action of this force, the surface seawater begins to flow along the shear stress of the wind. At the beginning of the flow, the viscous effect of the geostrophic deflection force and the lower static seawater on the upper moving seawater will hinder the flow of seawater. When the shear stress is balanced with the frictional force and the geostrophic deflection force, a stable ocean current is formed. Fixed currents and wind-driven currents are two classifications of wind-driven currents. Generally speaking, the wind currents caused by the widespread prevailing winds are called fixed currents, which are stable all the year round. They are also called drift or drift currents, and the currents formed by a shortterm weather process or gust are called wind-induced currents, which have great influence on navigation.

2) Ocean current

Ocean currents are caused by the difference of temperature, density, salinity and pressure between adjacent sea areas for a long time, or by the action of directional wind for a long time, which makes the sea water flow horizontally. Generally, the flow direction and velocity are basically unchanged in a certain period of time, so they are also called constant currents. The Kuroshio and Gulf Stream, two famous warm currents in the world, are formed by the difference of temperature and have great influence on the navigation of ships.

3) Tidal Stream, Tidal Current

Tidal changes are caused by the gravitational pull of celestial bodies. Tidal formation causes the horizontal movement of sea water caused by periodic fluctuations of sea water. This kind of current is called tidal current. Tidal currents are mainly divided into reciprocating and swirling currents. In the ocean, the tidal current is very small, mainly considering wind and sea currents. In offshore waters, the magnitude of tidal current can not be ignored, and there are strong tidal currents in narrow channels. The direction of reciprocating current is related to the fluctuation of tide. The average calculation formula for maximum velocity of flow:

maximum velocity of flow $=1 / 2$ (maximum velocity of Spring+maximum velocity of Neap)

$\approx 3 / 4$ maximum velocity of Spring

$$
\approx 3 / 2 \text { maximum velocity of Neap }
$$

Due to the influence of many factors, such as ocean shape, depth, bottom friction and ocean density stratification, the actual ocean tidal currents are very complex. Not only the tidal currents at different locations are different, but also the velocities and directions in different water layers at the same location are often quite different.

\subsubsection{The current effects on ship navigation}

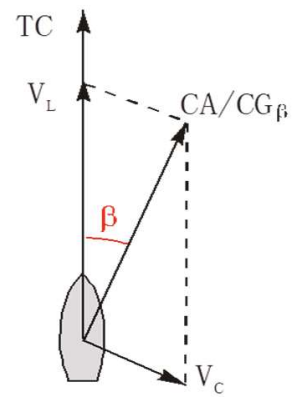

Figure 3. Drift angle Diagram. 
The direction of the current is different from the direction of the wind. It refers to the direction of the current. When the direction of the current is not collinear with the ship's first and last lines, the pressure difference angle beta will be generated (as shown in Figure 3). The current makes the pressure difference angle of the ship's heading right deflection positive and the pressure difference angle of the ship's heading left deflection negative.

The formula is expressed as:

$$
\mathrm{CG}(\mathrm{CA})=\mathrm{TC} \pm \beta
$$

In the formula:

$\mathrm{CG}(\mathrm{CA})$ _ course made good(course of advance);

$\mathrm{TC}$ - ture course

$\beta$ - drift angle

\subsection{Wind and current factors affecting ship navigation}

The relationship between ship's track direction and true course mainly depends on the combined action of the leeway and drift angle $\gamma: \gamma=\alpha+\beta$, and the positive and negative wind-current pressure difference depends on the result of the combined action of the leeway and drift angle. The relationship between CG and TC under the combined action of wind and current is as follows: $\mathrm{CG}=\mathrm{TC}+\gamma$.

\section{Measurement of leeway and drift angle in track plotting}

There are many methods to measure the leeway and drift angle in track plotting, but the principle is unified. They are all determined according to the following formulas: $\gamma=\mathrm{CG}-\mathrm{TC}$, Therefore, the key to determine the wind pressure difference is to find the $\mathrm{CG}$ and the TC, which can be obtained directly through the correction of the compass difference on the compass.

\subsection{Continuous observation and location method}

Figure. 4 shows that in a certain period of time, 3-5 observation positions are measured, and each observation position is connected by a "straight line", which is a track line (CG), and the angle between the track line and the true course is the wind pressure difference.

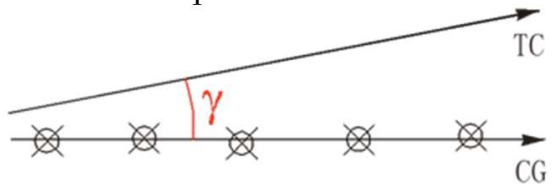

Figure 4. Continuous Observation and Location Method.

\subsection{Range navigation survey method}

As shown in Fig. 5, a pair of overlapping marks is selected to steer the ship along the overlapping mark line. At this time, the overlapping mark azimuth line is the measured track direction (CG). The difference between the overlapping mark and the true course is the wind pressure difference.

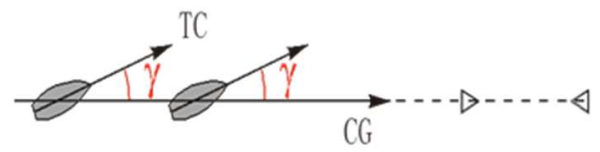

Figure. 5 Range navigation survey method

\subsection{Radar observation and measurement method}

Fig. 6 uses the relative upward motion of the bow to observe the echo a of something. Fig. 6 shows that the echo of the object is in a straight line, and adjusts the electronic azimuth line of radar so that they are parallel to the echo of the object. Then the angle between the electronic azimuth line and the bow line is the windcurrent pressure difference.

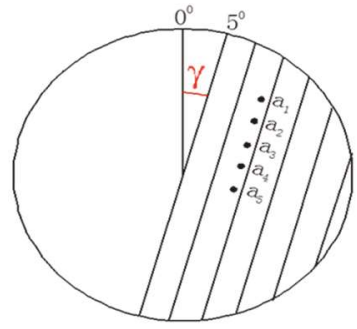

Figure 6. Radar Observation and Measurement Method.

\subsection{Method of Minimum Distance Bearing and Beam Bearing of Object}

As shown in Fig. 7:

$\because \mathrm{TB}_{\perp}=\mathrm{TC}+90^{\circ}$

$\mathrm{TBmin}=\mathrm{CG}+90^{\circ}$

$\therefore \mathrm{TBmin}-\mathrm{TB}_{\perp}=\mathrm{CG}-\mathrm{TC}=\gamma$

$\gamma=\mathrm{TBmin}-\mathrm{TB}$

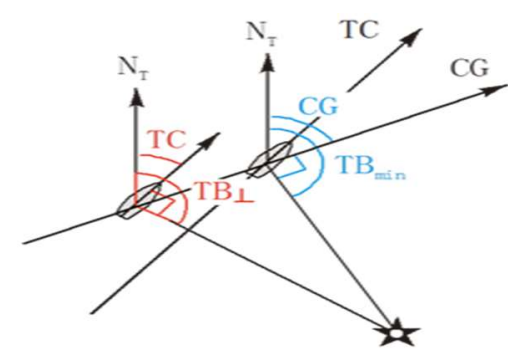

Figure 7. Method of Minimum Distance Bearing and Beam Bearing of Object.

Examples: A vessel $\mathrm{TC}=265^{\circ}$. The true bearing and distance of object are measured by radar observation method. The leeway and drift angle $(\gamma)$ and track direction (CG)are calculated in Table 1 below.

Table 1. Bearing and Distance.

\begin{tabular}{|c|c|c|c|c|c|c|c|c|c|}
\hline TB & $350^{\circ}$ & $355^{\circ}$ & $000^{\circ}$ & $003^{\circ}$ & $005^{\circ}$ & $008^{\circ}$ & $012^{\circ}$ & $015^{\circ}$ & $018^{\circ}$ \\
\hline D & $6.5^{\prime}$ & $6.3^{\prime}$ & $6.1^{\prime}$ & $6.0^{\prime}$ & $5.9^{\prime}$ & $5.8^{\prime}$ & $5.7^{\prime}$ & $5.8^{\prime}$ & $5.9^{\prime}$ \\
\hline
\end{tabular}

Solution: Observed results show that

$\mathrm{TBmin}=012^{\circ}$,

$\mathrm{TB}_{\perp}=\mathrm{TC}+90^{\circ}=265^{\circ}+90^{\circ}=355^{\circ}$

$\gamma=\mathrm{TBmin}-\mathrm{TB}_{\perp}=012^{\circ}-355^{\circ}=017^{\circ}$

$\mathrm{CG}=\mathrm{TC}+\gamma=265^{\circ}+17^{\circ}=282^{\circ}$ 


\section{Concluding remarks}

The accuracy of leeway and drift angle is directly related to the accuracy of ship track estimation. A deck officer should choose suitable and practical measurement methods according to the equipment condition of the ship and the natural environment of the navigation area, so as to make the measurement of leeway and drift angle accurate, fast and simple.

\section{References}

1. F. PCoenen, G. R. Smeaton and A. G. Bole. Knowledge-based collision avoidance.

2. M·ihiash, et al: Basic Research on a Collision Avoidance System using Neural Networks, Proceedings IAIN91, 1991.
3. M·Iniash, etal: Building Method and Application of Neural Network System for Navigation, Lateral Symposium of Sino-Japanese Navigation Institute, 1992

4. James, M.K.: Modeling the decision in computer simulation of ship navigation. Journal of Nvaigation, $39,32$.

5. H. IWASAKI and K. HARA, A Fuzzy Reasoning Model to Decide the Collision Avoidance Action, The Journal of Japan Institute of Nvaigation, Vol, 75, SeP, 1986.

6. K. HASEGAWA and A. KOUZKI, Automatic Collision Avoidance System for Ship Using Fuzzy Control, The Journal of Japan Institute of Navigation, Vol, 205, June, 1987. 